IMAGE FOCUS

\title{
Percutaneous closure of an acquired Gerbode defect
}

\author{
Amita Singh, Anuj Mediratta, Eric Kruse, Roberto M. Lang*, and Atman Shah
}

University of Chicago Medical Center, 5841 S. Maryland Avenue, Chicago, IL 60637, USA

*Corresponding author. E-mail: roberto.lang@medicine.bsd.uchicago.edu; rlang@bsd.uchicago.edu; rlang@medicine.bsd.uchicago.edu

An 89-year-old woman with history of bioprosthetic aortic valve replacement presented with worsening exertional dyspnoea. A transthoracic echocardiogram demonstrated the presence of a fistula between the left ventricle (LV) and right atrium (RA), consistent with an acquired Gerbode defect. Given her reluctance to pursue repeat surgery, a percutaneous approach for definitive closure was performed.

With peri-procedural transoesophageal echocardiography (TEE), systolic flow between the LVOT and RA was visualized. (Panel A; see Supplementary data online, Video S1) Using 3D image acquisition, the defect was characterized as circular in shape, measuring $9 \mathrm{~mm}$ in diameter. (Panel B; see Supplementary data online, Video S2) Under realtime 3D TEE, the defect was crossed with a wire from a transapical approach, and a $8 \mathrm{~mm}$ muscular VSD occluder was positioned across the defect. In-
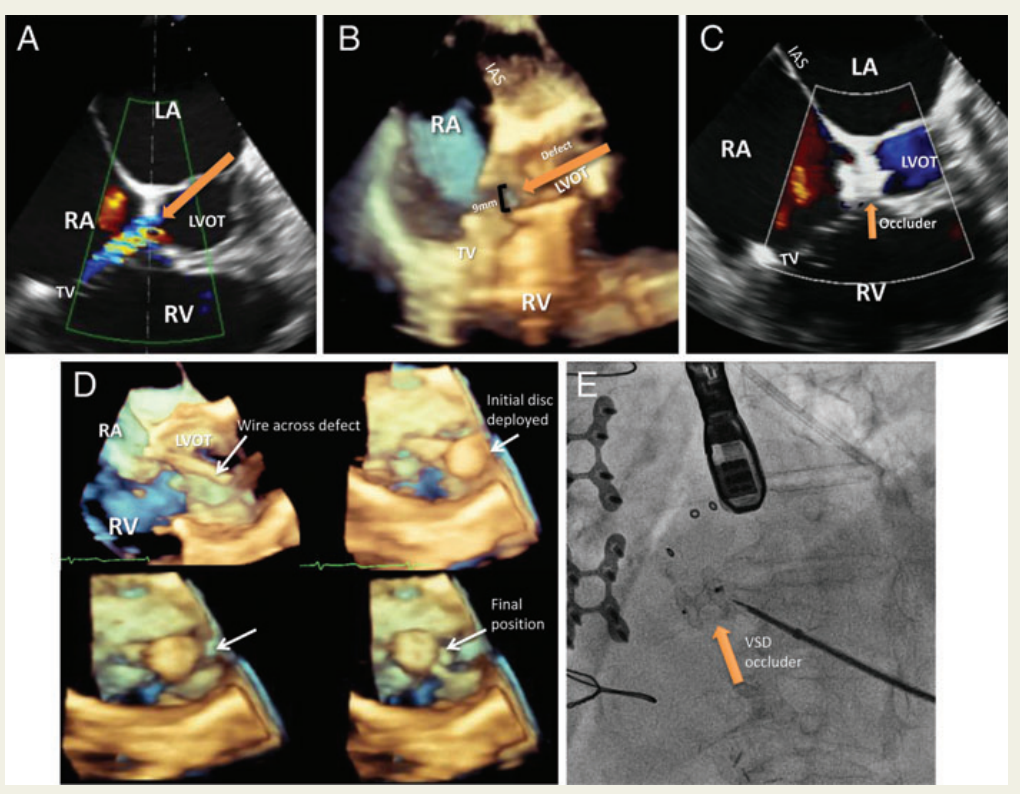
terrogation of the position of the occluder with $3 \mathrm{D}$ imaging was then performed and demonstrated sparing of the adjacent tricuspid and aortic valve apparatus. The occluder was then deployed with no residual flow detected (Panels C-E; Supplementary data online, Videos S3 and S4).

Classically described as congenital lesions, Gerbode defects can also be seen following aortic valve replacement. Overloading of the pulmonary vasculature occurs due to shunting from the high pressure LV to the low pressure RA, leading to symptoms of dyspnoea. Closure using occluder devices is complicated by the proximity of critical structures, including the tricuspid valve and AV nodal conduction system. Concomitant use of intraprocedural TEE facilitates appropriate sizing of the defect and safe deployment of the device, sparing the surrounding structures to ensure optimal procedural success.

Supplementary data are available at European Heart Journal-Cardiovascular Imaging online.

Published on behalf of the European Society of Cardiology. All rights reserved. (c) The Author 2016. For permissions please email: journals.permissions@oup.com. 